\title{
LMI-Based Design of LMS Adaptive Array Antenna Systems
}

\author{
Yuji Wakasa, Keiichi Nakashima and Kanya Tanaka \\ Department of Electrical and Electronic Engineering \\ Faculty of Engineering, Yamaguchi University \\ 2-16-1 Tokiwadai, Ube 755-8611, JAPAN \\ \{wakasa, ktanaka\}@eee.yamaguchi-u.ac.jp
}

\begin{abstract}
Recently, adaptive arrays have been studied in order to improve communication quality of mobile communication systems. The least mean square algorithm is one of techniques which can be applied to adaptive arrays. However, this method does not sufficiently exploit the degree of freedom of the step parameter of the recursive rule in the case where a priori information on the input signal is available. This paper proposes a design method of the step parameter such that the convergence of the recursive rule is guaranteed against the uncertainty of the input signal. The design problem of the step parameter is reduced to a semidefinite program which is efficiently solvable.
\end{abstract}

\section{Introduction}

Recently, adaptive array antenna systems have been studied in order to improve communication quality of mobile communication systems [5]. The adaptive array antenna system consists of multiple antenna elements whose weights can be adjusted to form directivity of the whole antenna system. To adjust the weights, the least mean square (LMS) algorithm is effective because of its computational simplicity and easy implementation. The standard LMS algorithm can be used without information on the input signal.

On the other hand, in the case where a priori information on the input signal is available, an LMS-type algorithm has been proposed such that the performance can be improved by exploiting the degree of freedom of the step parameter in the recursive rule [6].

In this paper, we propose a design method of LMS adaptive array antenna systems based on the design method in [6]. As a result, we show that the proposed algorithm gives better performance than the conventional algorithm from the viewpoint of convergence, error variance and robustness.

Notation: For a complex matrix $A, A^{T}, \bar{A}$ and $A^{*}$ stand for the transpose, complex conjugate and complex conjugate transpose of $A$, respectively.

\section{LMS adaptive array antenna}

Consider an adaptive array antenna system with $N$ array elements as shown in Figure 1.

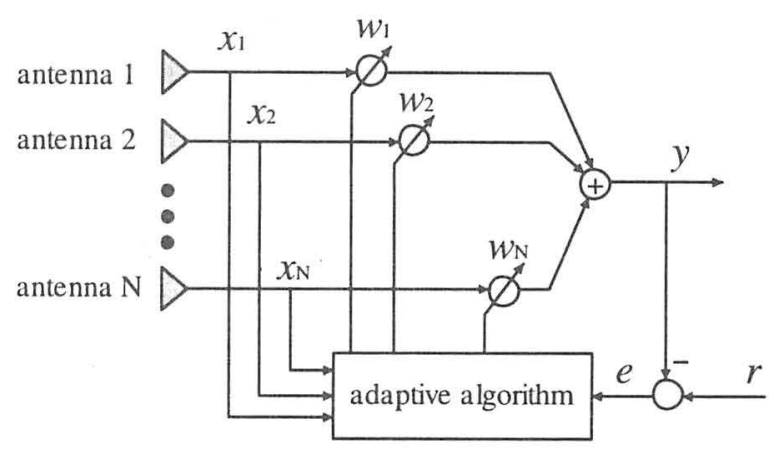

Figure 1: Adaptive array antenna system with $N$ antenna elements.

The purpose of the adaptive array antenna system is to automatically null out unknown interference and retain the desired signal by adjusting the weights of array elements. To this end, the weight vector $w \in \mathbb{C}^{N}$ is updated by minimizing the error signal $e$ between the reference signal $r$ and the array output $y$. The error signal $e$ to be minimized is expressed as follows:

$$
e(m)=r(m)-y(m)=r(m)-\boldsymbol{w}^{*} \boldsymbol{x}(m),
$$


where $\boldsymbol{x} \in \mathbb{C}^{N}$ is the vector of the received (input) signals at the antenna elements.

The recursive rule of the weights in the LMS algorithm is derived from a stochastic steepest descent method for minimizing the performance in$\operatorname{dex} J=E\left[|e|^{2}\right]$ as follows:

$$
\boldsymbol{w}(m+1)=\boldsymbol{w}(m)+K \boldsymbol{x}(m) \bar{e}(m),
$$

where $K$ is a step parameter conventionally given by $K=\mu I(\mu \in \mathbb{R}, \mu>0)$. In [6], it is shown that the algorithm with a more general diagonal matrix $K$ instead of $K=\mu I$ gives better performance at the same computational complexity.

Now, letting $\boldsymbol{w}_{\text {opt }}$ denote the optimal weight and subtracting $\boldsymbol{w}_{\text {opt }}$ from both sides of the recursive rule (1), we obtain

$$
\begin{aligned}
& \boldsymbol{w}(m+1)-\boldsymbol{w}_{\mathrm{opt}} \\
& \quad=\boldsymbol{w}(m)+K \boldsymbol{x}(m) \bar{e}(m)-\boldsymbol{w}_{\mathrm{opt}} \\
& \quad=\left(I-K \boldsymbol{x}(m) \boldsymbol{x}^{*}(m)\right)\left(\boldsymbol{w}(m)-\boldsymbol{w}_{\mathrm{opt}}\right) .
\end{aligned}
$$

We here make the following assumption:

Assumption 1 The weight vector $\boldsymbol{w}(m)$ changes more slowly than $\boldsymbol{x}(\mathrm{m})$ so that the following approximation holds:

$$
\begin{aligned}
& E\left[\left(I-K \boldsymbol{x}(m) \boldsymbol{x}^{*}(m)\right)\left(\boldsymbol{w}(m)-\boldsymbol{w}_{\mathrm{opt}}\right)\right] \\
& \quad=E\left[I-K \boldsymbol{x}(m) \boldsymbol{x}^{*}(m)\right] E\left[\boldsymbol{w}(m)-\boldsymbol{w}_{\mathrm{opt}}\right]
\end{aligned}
$$

This assumption is often used in analysis of the LMS algorithm [4]. Under the above assumption, taking the expected values of both sides of (2), we obtain

$E\left[\boldsymbol{w}(m+1)-\boldsymbol{w}_{\mathrm{opt}}\right]=(I-K R) E\left[\boldsymbol{w}(m)-\boldsymbol{w}_{\mathrm{opt}}\right]$, where $R:=E\left[\boldsymbol{x}(m) \boldsymbol{x}^{*}(m)\right]$ is the autocorrelation matrix of the input. Therefore, we conclude that $\lim _{m \rightarrow \infty} E[w(m)]=w_{\text {opt }}$ holds if and only if

$$
\left|\lambda_{i}(I-K R)\right|<1 \text { for all } i,
$$

where $\lambda_{i}(A)$ denotes the eigenvalue of a matrix $A$.

\section{Design of the recursive rule of the LMS algorithm}

In general, it is impossible to have the complete knowledge on the statistical information of the input of the system such as the autocorrelation matrix $R$. However, we can estimate such information to a certain extent, in the array antenna systems where the arrival direction of the input is limited within a certain range.

In this section, we design the step parameter $K \in \mathbb{C}^{N \times N}$ in the recursive rule (1) such that stability and performance of the algorithm are guaranteed even when the autocorrelation matrix of the input includes uncertainty as follows:

$$
R=\hat{R}+\Delta,
$$

where $\hat{R}$ is a nominal autocorrelation matrix and $\Delta$ is an uncertainty belonging to the following set:

$$
\Delta(\gamma):=\left\{\Delta \in \mathbb{C}^{N \times N}: \Delta=\Delta^{*},\|\Delta\| \leq \gamma\right\} .
$$

Here, $\|A\|$ denotes the largest singular value of a matrix $A$. Note that the size of uncertainty is determined by the parameter $\gamma>0$. In this section, we do not restrict the structure of $K \in \mathbb{C}^{N \times N}$, while the structure of $K$ in the conventional LMS algorithm is $K=\mu I, \mu \in \mathbb{R}$.

As in the previous section, we assume as follows:

Assumption 2 The weight vector $\boldsymbol{w}(m)$ changes more slowly than $\boldsymbol{x}(m)$ so that the following approximation holds for any $\Delta \in \Delta(\gamma)$ :

$$
\begin{aligned}
& E\left[\boldsymbol{w}(m+1)-\boldsymbol{w}_{\mathrm{opt}}\right] \\
& \quad=(I-K(\hat{R}+\Delta)) E\left[\boldsymbol{w}(m)-\boldsymbol{w}_{\mathrm{opt}}\right] .
\end{aligned}
$$

Then, $\lim _{m \rightarrow \infty} E[\boldsymbol{w}(m)]=\boldsymbol{w}_{\text {opt }}$ holds for any $\Delta \epsilon$ $\Delta(\gamma)$ if and only if

$$
\left|\lambda_{i}(I-K(\hat{R}+\Delta))\right|<1 \text { for all } i .
$$

Since the left side of (3)

$$
\max _{\Delta \in \Delta(\gamma)} \max _{i}\left|\lambda_{i}(I-K(\hat{R}+\Delta))\right|
$$

denotes the decay rate of $E\left[\boldsymbol{w}(m)-\boldsymbol{w}_{\text {opt }}\right]$, the coefficient $w(m)$ converges fast when (4) is small. In connection with this fact, the following lemma holds [1].

Lemma 1 For a matrix $A \in \mathbb{C}^{N \times N}$,

$$
\max _{i}\left|\lambda_{i}(A)\right|<\rho
$$

holds if and only if there exists an Hermitian matrix $P$ such that

$$
A^{*} P A-\rho^{2} P<0, P>0 .
$$


From Lemma 1 and Schur complement [1], it follows that $\boldsymbol{w}(m)$ converges if and only if there exist an Hermitian matrix $P$ and $\rho^{2} \leq 1$ such that

$$
\left[\begin{array}{cc}
\rho^{2} P & (I-K(\hat{R}+\Delta))^{*} P \\
P(I-K(\hat{R}+\Delta)) & P
\end{array}\right]>0,
$$

for all $\Delta \in \Delta(\gamma)$.

For this matrix inequality with uncertainty, we can derive a matrix inequality without uncertainty by using the following lemma [2].

Lemma 2 Let $S_{1}=S_{1}^{*}, S_{2}, S_{3}$ and $S_{4}$ be complex matrices of appropriate size. We have $\operatorname{det}(I-$ $\left.S_{4} \Delta\right) \neq 0$ and

$S_{1}+S_{2} \Delta\left(I-S_{4} \Delta\right)^{-1} S_{3}+S_{3}^{*}\left(\left(I-S_{4} \Delta\right)^{*}\right)^{-1} \Delta^{*} S_{2}^{*}>0$

for all $\Delta \in \Delta(\gamma)$, if and only if $\left\|S_{4}\right\|<1 / \gamma$, and there exists a scalar $\tau>0$ such that

$$
\left[\begin{array}{cc}
S_{1}-\tau \gamma^{2} S_{2} S_{2}^{*} & S_{3}^{*}-\tau \gamma^{2} S_{2} S_{4}^{*} \\
S_{3}-\tau \gamma^{2} S_{4} S_{2}^{*} & \tau\left(I-\gamma^{2} S_{4} S_{4}^{*}\right)
\end{array}\right]>0 .
$$

Proof This lemma can be proven in the same manner as Lemma 2.2 in [2] by taking account of the differences in the inequality sign and the complex matrices.

We obtain the following theorem which is useful for designing $K$ in the recursive rule.

Theorem 1 Suppose that Assumption 2 holds. Then, $\lim _{m \rightarrow \infty} E[\boldsymbol{w}(m)]=\boldsymbol{w}_{\text {opt }}$ holds for the recursive rule (1) if there exist an Hermitian matrix $P$, real scalars $\tau$ and $\rho^{2} \leq 1$ such that

$$
\left[\begin{array}{ccc}
\rho^{2} P-\tau \gamma^{2} I & (I-K \hat{R})^{*} P & 0 \\
P(I-K \hat{R}) & P & -P K \\
0 & -K^{*} P & \tau I
\end{array}\right]>0
$$

\section{Proof Let}

$$
\begin{gathered}
S_{1}:=\left[\begin{array}{cc}
\rho^{2} P & (I-K \hat{R})^{*} P \\
P(I-K \hat{R}) & P
\end{array}\right], \\
S_{2}:=\left[\begin{array}{l}
I \\
0
\end{array}\right], S_{3}:=\left[\begin{array}{ll}
0 & \left.-K^{*} P\right] \text { and } S_{4}:=0 .
\end{array}\right.
\end{gathered}
$$

Then, applying Lemma 2 and taking notice of $\Delta=$ $\Delta^{*}$, we obtain the results.

From Theorem 1, we can design the recursive rule with fast convergence by finding $P, K$, and $\tau$ satisfying (5) for a small positive scalar $\rho \leq 1$. However, since it is difficult to numerically solve (5), we introduce a variable $H \in \mathbb{C}^{N \times N}, H:=$ $P K$ and exploit the robust control approach in [1]. Namely, we design $K=P^{-1} H$ by computing an Hermitian matrix $P$ and a complex matrix $H$ such that

$$
\left[\begin{array}{ccc}
\rho^{2} P-\gamma^{2} I & P-\hat{R}^{*} H^{*} & 0 \\
P-H \hat{R} & P & -H \\
0 & -H^{*} & I
\end{array}\right]>0,
$$

where we normalize $\tau$ as $\tau:=1$. Inequality (6) is a complex linear matrix inequality (LMI), and therefore, we must convert this LMI into an equivalent real LMI in order to use recently developed tools based on interior-point methods such as [3]. To this end, we use the following lemma.

Lemma 3 Let $A$ and $B$ be real matrices. Then an Hermitian matrix $A+B j$ is positive definite if and only if a real symmetric matrix

$$
\left[\begin{array}{cc}
A & B \\
-B & A
\end{array}\right]
$$

is positive definite.

Now, defining $P=P_{r}+P_{i} j, H=H_{r}+H_{i} j$ and $\hat{R}=\hat{R}_{r}+\hat{R}_{i} j$ where $P_{r}, P_{i}, H_{r}, H_{i}, \hat{R}_{r}$ and $\hat{R}_{i}$ are all real matrices, we obtain the following equivalent real LMI for (6) from lemma 3 :

$$
\left[\begin{array}{cc}
A & B \\
-B & A
\end{array}\right]>0
$$

where

$A=\left[\begin{array}{ccc}\rho^{2} P_{r}-\gamma^{2} I & P_{r}-\hat{R}_{r}^{T} H_{r}^{T}+\hat{R}_{i}^{T} H_{i}^{T} & 0 \\ P_{r}-H_{r} \hat{R}_{r}+H_{i} \hat{R}_{i} & P_{r} & -H_{r} \\ 0 & -H_{r}^{T} & I\end{array}\right]$

$B=\left[\begin{array}{ccc}\rho^{2} P_{i} & P_{i}+\hat{R}_{r}^{T} H_{i}^{T}+\hat{R}_{i}^{T} H_{r}^{T} & 0 \\ P_{i}-H_{r} \hat{R}_{i}-H_{i} \hat{R}_{r} & P_{i} & -H_{i} \\ 0 & H_{i}^{T} & 0\end{array}\right]$.

Assumption 2 contradicts the design objective of the fast convergence of $\boldsymbol{w}(m)$ because it requires that $w(m)$ change slowly in comparison with $x(m)$. It should be noted that there exists such a tradeoff in designing the recursive rule as in [6]. In order to take account of this tradeoff, we reduce the values of $\left\|P^{-1}\right\|$ and $\|H\|$ so that $\boldsymbol{w}(m)$ is 
moderately updated since the following inequality holds from (1):

$$
\begin{aligned}
\|\boldsymbol{w}(m+1)-\boldsymbol{w}(m)\| & \leq\|K\|\|\boldsymbol{x}(m) \bar{e}(m)\| \\
& \leq\left\|P^{-1}\right\|\|H\|\|\boldsymbol{x}(m) \bar{e}(m)\| .
\end{aligned}
$$

From Schur complement [1], the necessary and sufficient conditions for $\left\|P^{-1}\right\| \leq \alpha$ and $\|H\| \leq \beta$ are as follows:

$$
\begin{aligned}
& \left\|P^{-1}\right\| \leq \alpha \Leftrightarrow\left[\begin{array}{ccc}
P_{r}-\frac{1}{\alpha} I & P_{i} \\
-P_{i} & P_{r}-\frac{1}{\alpha} I
\end{array}\right] \geq 0 \\
& \|H\| \leq \beta \Leftrightarrow\left[\begin{array}{cccc}
\beta^{2} I & H_{r}^{T} & 0 & -H_{i}^{T} \\
H_{r} & I & H_{i} & 0 \\
0 & H_{i}^{T} & \beta^{2} I & H_{r}^{T} \\
-H_{i} & 0 & H_{r} & I
\end{array}\right] \geq 0 .
\end{aligned}
$$

To sum up, we can design $K=P^{-1} H$ by solving the following semidefinite programming problem: for given design parameters $\rho, \gamma$ and $\alpha$, minimize $\beta^{2}$ subject to LMIs (8), (9) and (10).

\section{Numerical example}

Consider an adaptive array antenna system with 2 antenna elements. The desired signal $s(m)$ and the undesired signal $u(m)$ achieve the antenna in the directions $\theta_{s}=60^{\circ}$ and $\theta_{u}=-30^{\circ}$, respectively, from the broadside direction as shown in Figure 2. Suppose that the interval between the array elements is the half of the wavelength $(\lambda / 2)$ of the carrier wave, and that the reference signal $r(m)$ is the same as the desired signal $s(m)$. Then the sampled input signal vector is shown by

$$
x(m)=s(m)\left[\begin{array}{c}
1 \\
\exp \left(-j \frac{2 \pi}{\lambda} d \sin \left(\frac{\pi}{3}\right)\right)
\end{array}\right]
$$

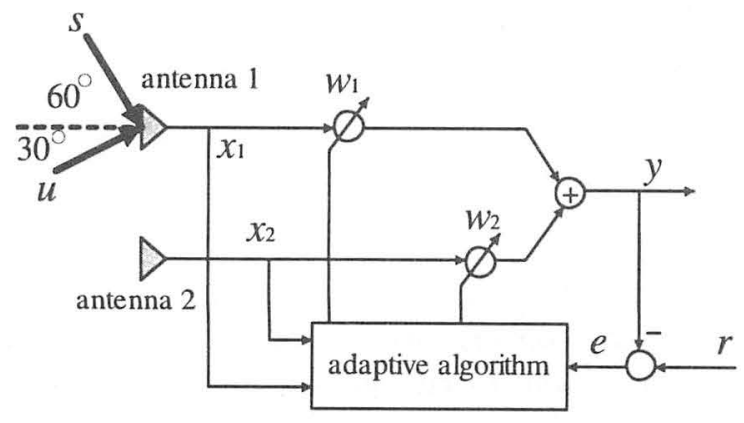

Figure 2: Adaptive antenna array system with 2 antenna elements.

$$
\begin{aligned}
& +u(m)\left[\exp \left(-j \frac{2 \pi}{\lambda} d \sin \left(-\frac{\pi}{6}\right)\right)\right] \\
& +\left[\begin{array}{c}
n_{1}(m) \\
n_{2}(m)
\end{array}\right],
\end{aligned}
$$

where $n_{1}$ and $n_{2}$ are the internal noises of the array element 1 and 2, respectively, and $s(m)$ and $u(m)$ are randomly generated with $\phi_{s}(m), \phi_{u}(m) \in$ $[0,2 \pi)$ by

$$
\begin{aligned}
& s(m)=\exp \left(j \phi_{s}(m)\right), \\
& u(m)=\exp \left(j \phi_{u}(m)\right) .
\end{aligned}
$$

The numerical example is carried out for the initial weight $w(0)=[1,0]^{T}$ on MATLAB.

Generally, it is known that there is a tradeoff between the convergence time and the variance of $e(m)$ in steady-state. Figure 3 shows tradeoff curves by the conventional and proposed methods. From these tradeoff curves, we see that the convergence property and steady-state property by the proposed method are superior to those by the conventional one.

To compare the convergence speeds of the conventional and proposed algorithms, the design parameters are chosen so that the error variances are almost the same in the two algorithms. The parameters for the proposed and conventional methods are chosen as $\rho=0.96$ and $\mu=0.05$, respectively. Figure 4 shows the errors $E[|e(m)|]$ by the proposed and conventional methods. 100 trials are averaged for the errors. We see that the proposed algorithm converges faster than the conventional one.

Figures 5 and 6 show the array responses for various directions of the signal by the proposed $(\rho=0.95)$ and conventional $(\mu=0.05)$ methods, respectively. As we expect, the array responses for the direction of the desired signal are high while those for the undesired signal are low. It is seen that the proposed method gives a better property of the array response than the conventional one.

\section{Conclusion}

In this paper, we have proposed a design method of LMS adaptive array antenna systems by applying the design method in [6]. As a result, we have shown that the proposed algorithm gives better performance than the conventional algorithm from the viewpoint of convergence and error variance. 


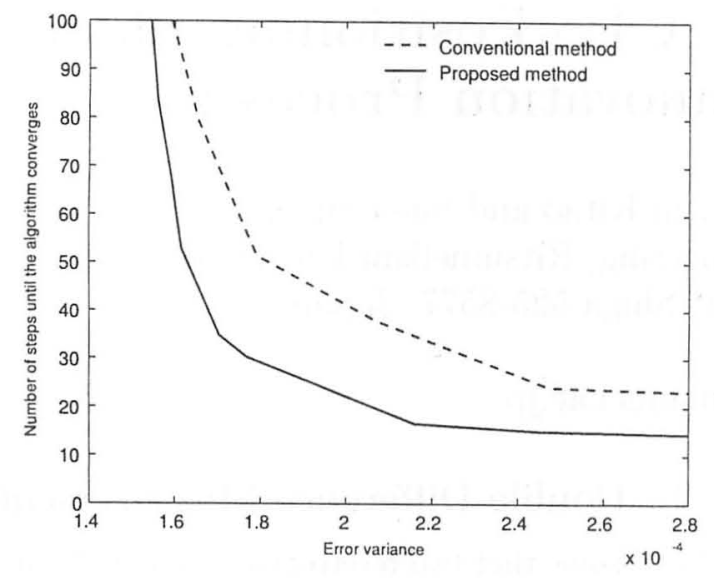

Figure 3: Tradeoff curves between the convergence speed and the error variance.

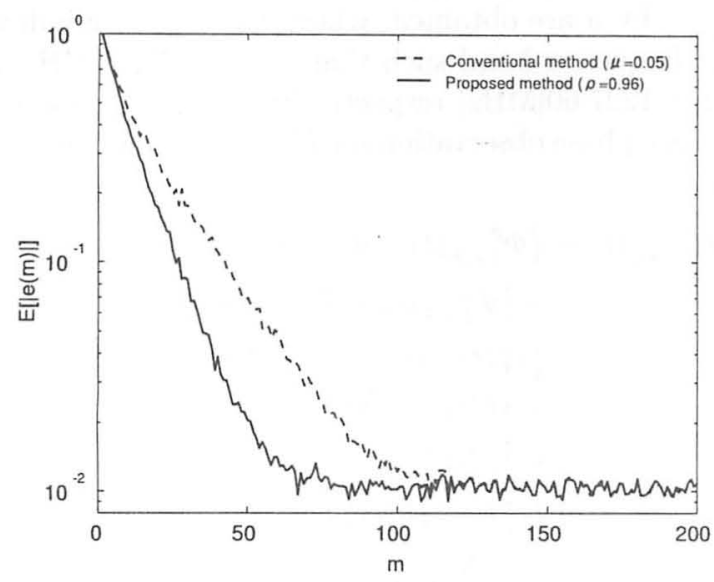

Figure 4: Convergence property.

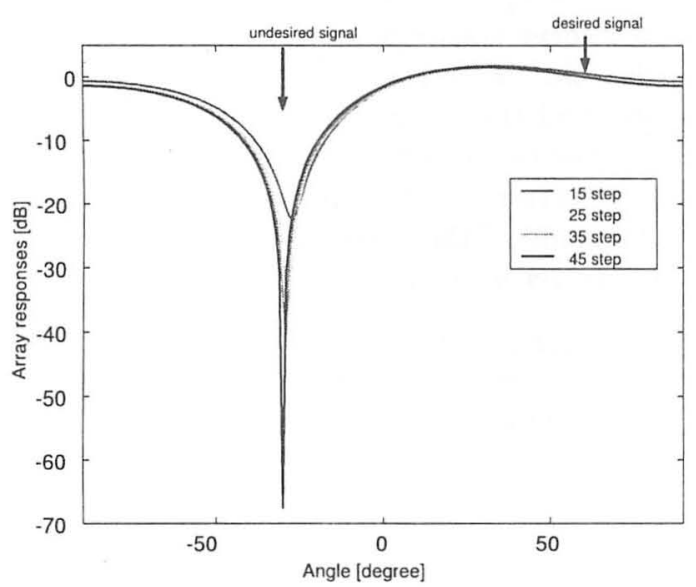

Figure 5: Array responses by the proposed method.

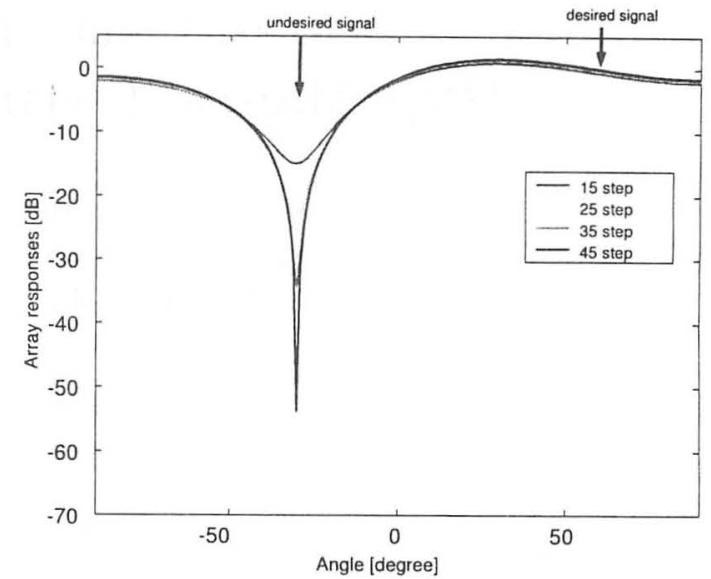

Figure 6: Array responses by the conventional method.

\section{References}

[1] S. Boyd, L. El Ghaoui, E. Feron and V. Balakrishnan, Linear Matrix Inequalities in System and Control Theory, Philadelphia, PA: SIAM, 1994.

[2] L. El Ghaoui and H. Lebret, "Robust solutions to least-squares problems with uncertain data," SIAM J. Matrix Analysis and Applications, vol. 18, no. 4, pp. 1035-1064, 1997.

[3] P. Gahinet, A. Nemirovski, A. J. Laub and M. Chilali, LMI Control Toolbox, For Use with MATLAB, The MathWorks, Inc., 1995.

[4] S. Haykin, Adaptive Filter Theory, 3rd ed., Upper Saddle River, NJ: Prentice-Hall, 1996.

[5] N. Kikuma, Adaptive Signal Processing with Array Antenna, Science and Technology Publishing Co., Inc., 1999 (in Japanese).

[6] Y. Wakasa, M. Hikita and Y. Yamamoto, "LMS adaptive filter design using semidefinite programming and its application to active noise control," Trans. ISCIE, vol. 16, no. 9, pp. 461-467, 2003 (in Japanese). 